\title{
Lidil
}

Revue de linguistique et de didactique des langues

$60 \mid 2019$

Langues des signes et genres discursifs

\section{Conversations spontanées en langue des signes de Belgique francophone (LSFB) : fonctions et usages de la répétition}

Spontaneous Conversations in French Belgian Sign Language (LSFB): Functions and Uses of Repetitions

Ingrid Notarrigo et Laurence Meurant

\section{OpenEdition}

Journals

Édition électronique

URL : http://journals.openedition.org/lidil/7002

DOI : 10.4000/lidil.7002

ISSN : 1960-6052

Éditeur

UGA Éditions/Université Grenoble Alpes

Édition imprimée

ISBN : 978-2-37747-164-5

ISSN : $1146-6480$

Référence électronique

Ingrid Notarrigo et Laurence Meurant, « Conversations spontanées en langue des signes de Belgique francophone (LSFB) : fonctions et usages de la répétition », Lidil [En ligne], 60 | 2019, mis en ligne le 01 novembre 2019, consulté le 14 avril 2020. URL : http://journals.openedition.org/lidil/7002 ; DOI : https://doi.org/10.4000/lidil.7002

Ce document a été généré automatiquement le 14 avril 2020

(C) Lidil 


\title{
Conversations spontanées en langue des signes de Belgique francophone (LSFB) : fonctions et usages de la répétition
}

\author{
Spontaneous Conversations in French Belgian Sign Language (LSFB): Functions \\ and Uses of Repetitions
}

Ingrid Notarrigo et Laurence Meurant

\section{Introduction}

1 Cet article décrit l'emploi de la répétition de signes dans des extraits de conversations en langue des signes de Belgique francophone (LSFB). Le phénomène de la répétition a précédemment été observé dans cette langue pour sa fonction morphologique de délimitation des unités (Meurant, 2010), pour son rôle dans la construction syntaxique et l'expression de l'alternance des points de vue (Meurant, 2008), ou encore pour sa fonction de marqueur de la reformulation (Meurant \& Sinte, 2016). La variété des fonctions identifiées en LSFB et des niveaux d'analyse impliqués (morphologie, syntaxe, énonciation, structuration et progression discursive) trouve un écho dans la littérature consacrée à d'autres langues des signes (LS) (Klima \& Bellugi, 1979; Börstell, 2011) et aux langues vocales (LV) (Frédéric, 1985 ; Tannen, 2007).

2 L'étude présentée ici propose une typologie des répétitions et une description globale de leur usage dans des conversations entre signeurs de la LSFB. Le corpus analysé comptabilise 30 minutes de vidéo, représentant les productions de 18 signeurs dans une tâche de conversation. Aucune distinction n'a été faite entre les répétitions qui semblent nécessaires grammaticalement et celles qui auraient un statut plus facultatif, ni entre les répétitions marquant une hésitation et celles qui apparaissent hors contexte d'hésitation. Les répétitions lexicales liées à la thématique de la discussion ont 
aussi été prises en compte. Ces choix ont été faits dans le but de disposer d'un aperçu complet du phénomène étudié.

\section{Approches de la répétition}

Parmi l'importante littérature consacrée aux phénomènes de répétition en $\mathrm{LV}$, on compte des travaux liés à l'étude de la répétition contigüe (ou quasi-contigüe) en contexte d'hésitation. En cherchant à distinguer les répétitions qui sont l'indice d'une disfluence de celles qui n'en sont pas, Candea (2000), Shriberg (1994) et Dister (2007) mettent en évidence différents types de répétitions, présentes à l'écrit comme à l'oral, qui relèvent de la syntaxe ou d'un effet oratoire. Parmi les cas de répétitions non disfluentes, Dister (2007, p. 118) mentionne les exemples suivants : nous nous sommes trompés (verbe pronominal); ça ne sert à rien d'être austère tout le temps tout le temps tout le temps (interprétation intensive); et puis alors un gâteau mais un gâteau gâteau pas un gâteau de glace (modification du sens avec une restriction); vous vous ne pouvez pas en dire autant (mise en évidence). Par ailleurs, d'autres occurrences aident à la planification de la pensée et soutiennent le travail de formulation à l'oral. Par exemple, dans ça va il a l'air de de te reconnaitre, la répétition de "de » ne porte aucune fonction syntaxique ou sémantique (Dister, 2007, p. 121). La littérature consacrée à l'hésitation dans les LS reste encore aujourd'hui peu abondante, mais la répétition contigüe y est identifiée comme un marqueur de disfluence. Elle permet de combler un moment d'hésitation, de s'auto-corriger (Nicodemus, 2011 ; Hohenberger \& Leuninger, 2012), ou de relancer l'énoncé après une parenthèse (Risler, 2014).

De nombreux travaux portent ensuite sur la répétition (contigüe et non contigüe) envisagée comme un outil linguistique et conversationnel (Frédéric, 1985; Tannen, 2007). Les fonctions linguistiques relevées en LV trouvent leurs correspondants dans diverses LS. Ainsi, le dédoublement (doubling) d'un patron rythmique, la duplication (reduplication) flexionnelle ou dérivationnelle (Klima \& Bellugi, 1979; Bergman \& Dahl, 1994 ; Pfau \& Steinbach, 2006 ; Börstell, 2011 ; Herbert, 2012), la répétition (repetition) servant la structure et les relations syntaxiques (Vermeerbergen \& De Vriendt, 1994 ; Branchini et coll., 2013), la répétition sémantique au service de la progression du sens, de l'établissement de relations et de contrastes sémantiques (Kimmelman, 2014), et la répétition pragmatique liée à la planification et à l'organisation du propos et des points de vue dans le discours (Fischer \& Janis, 1990; Crasborn et coll., 2009; Kimmelman, 2014).

Enfin, une forme de répétition que nous appellerons «encadrante" fait l'objet de travaux tant en LV qu'en LS. Les termes répétés encadrent de façon symétrique un élément central, sur le modèle X Y X. Les costrutti-eco de Dovicchi (2010), typiques d'une variété de l'oral dialectal calabrais et sicilien, constituent un moyen de mettre en évidence un élément du discours en l'encadrant, ou de rétablir le discours après des moments de complexité liés à l'interaction (hésitation, réparation). La même structure de type XYX a été étudiée dans différentes LS, sous le nom de bracketing (Vermeerbergen \& De Vriendt, 1994), de doubling (Filipczak \& Mostowski, 2013; Kimmelman, 2014) ou de topic copying (Crasborn et coll., 2009), comme permettant de délimiter les constituants d'une unité syntaxique ou des unités syntaxiques entières, de jouer un rôle dans l'emphase ou la mise en relief d'informations, ou de lier sémantiquement plusieurs unités syntaxiques. 
6 Sur la base de ces descriptions en LV et en LS, et en s'appuyant sur l'observation de données de LSFB et de VGT (Vlaamse Gebarentaal), Notarrigo et coll. (2016) ont établi une typologie des formes et des fonctions des répétitions de signes manuels permettant de répertorier la plus large palette possible des occurrences rencontrées, sans à priori sur le caractère plus ou moins nécessaire, volontaire ou grammatical des répétitions. L'opérationnalité de cette typologie a été vérifiée par un test inter-annotateurs qui montre un bon taux d'accord sur le repérage des répétitions et sur leur qualification formelle et fonctionnelle (Notarrigo, 2017, p. 260-263) ${ }^{1}$. Cet article vise à présenter chacune des catégories de la typologie établie et à décrire leur usage au sein de conversations en LSFB.

\section{Répétition : délimitation du phénomène}

7 Une répétition comporte deux parties: le répétable et le(s) répété(s), c'est-à-dire l'élément qui sera répété et le/les élément(s) qui le répète(nt). Le terme de "répétition » désignera toujours l'intégralité du bloc formé par le répétable suivi de tous ses répétés (Candea, 2000, p. 315). Notons que le répétable peut être composé d'un ou de plusieurs éléments et que, dans le répété, la distance entre les signes et leur ordre d'apparition ne doit pas être nécessairement respectée.

8 Contrairement à Candea (2000, p. 28), nous ne nous limiterons pas aux répétitions constituées d'un répétable et d'un répété parfaitement identiques et contigus, dès lors que le propos ne se limite pas à la question de l'hésitation. Les répétitions du type de je l'ai vu euh je l'avais vu, une maison une belle maison, que Candea écarte, seront prises en compte ici. De plus, nous avons accepté une certaine variabilité entre la réalisation d'un répétable et celle de ses répétés. Par exemple, un répété peut être articulé de manière plus relâchée avec un mouvement plus court que le répétable, ou ne pas être exécuté avec le même arrangement des mains (p. ex. : répétable à une main, répété à deux mains), ou bien présenter une localisation différente dans l'espace, ou encore être accompagné d'une activation différente des articulateurs non manuels ${ }^{2}$ (Vermeerbergen \& De Vriendt, 1994 ; Kimmelman, 2014). Par contre, les phénomènes suivants sont exclus de l'analyse : les synonymes; les homophones; les gestes palm-ups (Kendon, 2004 ; Van Loon, 2012 ; Gabarró-López, 2017) ; les troncations, dans la mesure où elles ne présentent qu'une répétition partielle du signe; les buoys, ou maintiens d'une main dans la position du signe pendant que l'autre continue à signer (Liddell, 2003; Gabarró-López \& Meurant, 2014); la réduplication lexicale liée au processus de dérivation (Klima \& Bellugi, 1979) ; la réalisation simultanée d'un signe unimanuel par les deux mains, notamment pour exprimer la réciprocité (Pfau \& Steinbach, 2006; Meurant, 2008); la répétition d'un élément non manuel; la répétition d'un patron rythmique appliqué à des signes différents.

9 Les cas de réduplications internes dans le mouvement même d'un signe et des répétitions de signes pointés ont été partiellement pris en compte. Afin d'écarter les cas où elle est simplement définitoire de la forme lexicale et les cas de dérivation lexicale, la réduplication interne au signe a été prise en compte si le mouvement est répété trois fois, comme dans les exemples 1 et 5 ci-dessous (Pfau \& Steinbach, 2006), et/ou avec une répétition du mouvement des lèvres, et/ou avec un changement d'emplacement (Börstell, 2011). Les signes pointés ont été répertoriés si les occurrences 
répétées pouvaient être identifiées comme fonctionnant ensemble et si la répétition remplissait une fonction identifiable.

Nous considérons toute apparition multiple d'un signe au sein d'un même tour de parole comme un cas de répétition et nous en identifions le rôle, que ce soit au niveau grammatical, sémantique ou pragmatique. L'attention est donc portée sur de larges empans de discours (les tours de parole vont de 10 secondes à 2 minutes) et non uniquement sur des phénomènes locaux.

\section{Données et annotations}

11 Les productions de 18 signeurs, extraites du Corpus LSFB (Meurant, 2015), ont été analysées ${ }^{3}$. Pour chaque signeur, des extraits d'environ 2 minutes ont été sélectionnés au sein de conversations spontanées dont le sujet pouvait être : les variations au sein des usages de la LSFB (tâche 14), l'impact des émotions du signeur sur sa façon de signer (tâche 6), ou la notion de « bien signer » (tâche 5) ${ }^{4}$. Les plus longs tours de parole de chaque signeur ont été sélectionnés au sein de chaque tâche, en excluant tous les passages marqués par une interaction entre les deux participants ${ }^{5}$.

Tableau 1. - Données étudiées.

\begin{tabular}{|c|c|c|c|c|c|c|c|c|}
\hline Session & Signeur & Profil & Âge & Sexe & Tâches & $\begin{array}{l}\text { Temps } \\
\text { (sec.) }\end{array}$ & $\begin{array}{l}\text { Nombre de } \\
\text { signes }\end{array}$ & $\begin{array}{ll}\text { Nombre } & \text { de } \\
\text { répétitions } & \end{array}$ \\
\hline 6 & S013 & Natif & 30 & $\mathrm{~F}$ & 6 & 110,7 & 179 & 48 \\
\hline 8 & S017 & Natif & 30 & $\mathrm{H}$ & 14 & 99,7 & 209 & 73 \\
\hline 11 & S026 & Natif & 42 & $\mathrm{~F}$ & 14 & 146,25 & 396 & 94 \\
\hline 19 & S041 & Natif & 33 & $\mathrm{H}$ & 5 & 140,4 & 295 & 97 \\
\hline 37 & S075 & Natif & 33 & $\mathrm{H}$ & 14 & 130,8 & 286 & 78 \\
\hline 37 & S076 & Natif & 32 & $\mathrm{H}$ & 5 et 6 & 124,7 & 309 & 77 \\
\hline 3 & S007 & $\begin{array}{l}\text { Quasi- } \\
\text { natif }\end{array}$ & 40 & $\mathrm{H}$ & 6 & 196,3 & 385 & 85 \\
\hline 3 & S008 & $\begin{array}{l}\text { Quasi- } \\
\text { natif }\end{array}$ & 41 & $\mathrm{H}$ & 6 & 166,2 & 289 & 76 \\
\hline 16 & S036 & $\begin{array}{l}\text { Quasi- } \\
\text { natif }\end{array}$ & 29 & $\mathrm{~F}$ & 6 & 126,1 & 261 & 78 \\
\hline 16 & S037 & $\begin{array}{l}\text { Quasi- } \\
\text { natif }\end{array}$ & 29 & $\mathrm{H}$ & 14 & 131,8 & 282 & 95 \\
\hline 33 & S067 & $\begin{array}{l}\text { Quasi- } \\
\text { natif }\end{array}$ & 37 & $\mathrm{~F}$ & 14 & 113,1 & 234 & 67 \\
\hline
\end{tabular}




\begin{tabular}{|l|l|l|l|l|l|l|l|l|}
\hline 33 & S068 & $\begin{array}{l}\text { Quasi- } \\
\text { natif }\end{array}$ & 39 & F & 14 & 121,9 & 234 & 80 \\
\hline 23 & S005 & Tardif & 37 & F & 14 & 138,1 & 251 & 86 \\
\hline 24 & S049 & Tardif & 28 & F & 6 & 139,3 & 285 & 78 \\
\hline 24 & S050 & Tardif & 27 & F & 6 & 167,5 & 283 & 80 \\
\hline 30 & S061 & Tardif & 30 & F & 6 et 14 & 132,5 & 214 & 51 \\
\hline 34 & S069 & Tardif & 41 & F & 14 & 125,9 & 153 & 43 \\
\hline 34 & S070 & Tardif & 35 & H & 14 & 155,2 & 281 & 80 \\
\hline Total & & & & & & 2466,45 & 4826 & 1366 \\
\hline
\end{tabular}

Le protocole d'annotation des répétitions est issu d'une recherche collaborative sur l'étude des marques de fluence et de disfluence en LV et en LS (Crible et coll., 2019) et détaillé dans Notarrigo (2017, p. 253-260). Les vidéos ont été annotées dans le logiciel $\operatorname{ELAN}^{6}$ (Crasborn \& Sloetjes, 2008).

Pour des raisons pratiques liées à l'analyse quantitative des données, chaque répétition n'a reçu qu'une seule étiquette de fonction. En cas d'hésitation entre plusieurs fonctions, les trois principes suivants ont été appliqués: (1) donner la priorité au domaine grammatical ; (2) sélectionner la fonction de cohésion uniquement quand il n'y a aucune autre option; (3) donner l'avantage aux fonctions qui ne sont pas directement en lien avec le processus d'hésitation pour donner le bénéfice du doute au signeur.

\section{Typologie et résultats}

\subsection{Formes des répétitions}

D'un point de vue formel, trois types de répétitions sont distingués. Les répétitions contigües $(\mathrm{RC})$ présentent un répétable suivi directement de son ou de ses répétés, ou séparé d'eux par un élément comme une tenue, une pause, un geste de recherche lexicale ou encore par un palm-up (exemple 1). Les répétitions non contigües (RN) présentent un répétable séparé de son ou de ses répétés par au moins un autre signe (exemple 2).

(1)

PT:1 RAPPELER LONGTEMPS LONGTEMPS LONGTEMPS

'Je me souviens, c'était il y a très longtemps.'

(CLSFB_03_06, S008, 03:04.500-03:06) ${ }^{7}$

(2)

AVANT INTERDIRE LS INTERDIRE ÉCOLE INTERDIRE

‘Ça avait été interdit. La langue des signes a été interdite. Dans l'école, c'était interdit.'

(CLSFB_34_14, S069, 02:07-02:15) 
15 Parmi les RN, les répétitions encadrantes sont identifiées comme une catégorie à part $(\mathrm{RNe})$. Il s'agit des cas où le répétable et le répété sont disposés symétriquement de part et d'autre d'un élément central (composé d'un ou de plusieurs signes) de manière à ce que le répété ferme le segment que le répétable a ouvert, comme dans l'exemple 3.

(3)

PARENTS PT:POSS RÉGULIER ASSOCIATION RÉGULIER

'Mes parents vont régulièrement dans des associations [pour sourds].'

(CLSFB_11_14, S026, 02:53.800-02:55.600)

$16 \mathrm{Au}$ sein des données étudiées, 1366 répétitions ont été recensées sur un total de 30 minutes et de 4826 signes, ce qui correspond à une fréquence moyenne de 28,65 répétitions sur 100 signes $^{8}$. Les RN sont beaucoup plus fréquentes que les RC et que les RNe. En moyenne, on trouve 17,64 RN sur 100 signes, contre 5,74 RC et 5,26 RNe. Ces chiffres rejoignent ceux de Filipczak et Mostowski (2013) et de Kimmelman (2014).

\subsection{Fonctions des répétitions}

17 Du point de vue de leurs fonctions, les répétitions ont été classées en trois domaines (grammatical, sémantique et pragmatique), eux-mêmes divisés en sous-domaines. Le domaine grammatical recouvre les répétitions liées à la structure morpho-syntaxique : par exemple, les cas de flexion aspectuelle et de délimitation des constituants d'une unité syntaxique. Le domaine sémantique couvre les répétitions qui soutiennent le tissage $\mathrm{du}$ sens $\mathrm{du}$ discours, en particulier sa cohérence, sa chronologie et la progression de l'information. Le domaine pragmatique inclut les répétitions qui participent à l'organisation du discours, dans sa structuration énonciative et dans la gestion de l'interaction. Parmi toutes les répétitions identifiées, la moitié $(49,5 \%)$ ont une fonction pragmatique, un quart $(24,5 \%)$ relèvent du domaine sémantique, contre $18 \%$ qui concernent le domaine grammatical et $8 \%$ d'occurrences qui ne relèvent d'aucun de ces trois domaines.

Les fonctions grammaticales sont essentiellement remplies par des RC pour les fonctions portant sur le signe lui-même (cf. ci-dessous) et par des RN et des RNe pour celles qui portent sur l'unité syntaxique. Les fonctions sémantiques et pragmatiques sont en grande majorité remplies par les RN, avec quelques cas de RC et de RNe.

\subsubsection{Fonctions grammaticales}

Le domaine grammatical se subdivise en deux sous-domaines : le premier concerne les répétitions qui portent sur le signe lui-même et le second celles qui jouent un rôle au niveau de l'unité syntaxique. Chaque sous-domaine inclut ensuite une série de fonctions qui sont listées dans le tableau 2 (pour une présentation détaillée de chacune, voir Notarrigo, 2017, p. 220-253). Pour chaque sous-domaine, l'une ou l'autre des fonctions les plus fréquemment rencontrées dans nos données seront illustrées.

Tableau 2. - Typologie fonctionnelle - Domaine grammatical.

\begin{tabular}{|l|l|}
\hline Domaine grammatical \\
\hline Au niveau du signe & Au niveau de l'unité syntaxique \\
\hline
\end{tabular}




\begin{tabular}{|l|l|l|l|}
\hline Fonctions & Nombre d'occurrences & Fonctions & Nombre d'occurrences \\
\hline$<G:$ Pluriel> & 60 & $<G:$ Syntagme> & 47 \\
\hline$<G:$ Intensité> & 34 & $<G:$ Subordonnée> & 15 \\
\hline$<G:$ Durée> & 29 & $<G:$ Citation> & 12 \\
\hline$<G:$ Réitération> & 23 & $<G:$ Interrogative> & 11 \\
\hline$<G:$ Modalité> & 4 & & \\
\hline$<G:$ Réciprocité> & 2 & & \\
\hline$<G:$ Approximation> & 2 & Total & 85 \\
\hline Total & 165 & & \\
\hline
\end{tabular}

20 L'une des formes du pluriel en LSFB, comme dans d'autres LS, se fonde sur la répétition du signe (Klima \& Bellugi, 1979; Pfau \& Steinbach, 2006; Meurant, 2008; Herbert, 2012). L'exemple 4 en donne une illustration.

(4)

ÉTUDIER VOCABULAIRE PHRASE PHRASE PLUS MOT À-CÔTÉ DÉFINITION PLUS EXEMPLE EXEMPLE EXEMPLE

'Ils étudient le vocabulaire à l'intérieur de phrases. À côté du mot, il y a une définition et des exemples.'

(CLSFB_19_05, S041, 07:11-07:16.600)

21 La répétition d'un signe, généralement accompagnée d'une utilisation forte des éléments non manuels, peut aussi apporter un sens d'intensification (Klima \& Bellugi, 1979). C'est le cas de l'exemple 1 ci-dessus, mais aussi de l'exemple 5, où l'expression du visage renforce l'effet de la répétition dans l'expression de l'intensité de l'effort.

(5)

PT:1 RETOUR BIEN ENTRAINEMENT ENTRAINEMENT ENTRAINEMENT

'Une fois rétabli, j'ai recommencé les entrainements d'arrache-pied.'

(CLSFB_03_06, S008, 11:31-11:33)

Le second sous-domaine concerne les RNe impliquées dans la structuration grammaticale d'une unité syntaxique (Vermeerbergen \& De Vriendt, 1994 ; Branchini et coll., 2013), ainsi que les RNe qui lient deux unités syntaxiques entre elles par enchâssement (Hodge, 2013). Les cas les plus fréquemment rencontrés sont ceux où, comme dans l'exemple 6, la répétition borde les frontières internes d'un syntagme. Dans le cas de l'exemple 6, deux répétitions successives ( $\angle$ COCA...COCA $>$ et $<$ NOIR... NOIR>) délimitent chacune un syntagme dont le centre est BOIRE. D'après Vermeerbergen et De Vriendt (1994), ce genre de répétitions correspondrait à une actualisation des deux places possibles pour recevoir le terme répété au sein d'une unité syntaxique en langue des signes.

(6)

PARENTS ENTENDANT PT:DET COCA BOIRE COCA INCAPABLE NOIR BOIRE NOIR ENFANT PENSER C'EST COCA BOIRE NOIR

'Les parents entendants ne sont pas capables de dire à leur enfant sourd en langue des signes « coca ». À la place ils disent « la boisson noire ». L'enfant sait que « la 
boisson noire ", ça veut dire « coca »'

(CLSFB_33_14, S067, 02:39.800-02:44.800)

L'encadrement d'une subordonnée est, dans ce sous-domaine, la seconde fonction la plus représentée. La répétition porte alors sur un verbe d'opinion, de déclaration, de perception, ou sur un introducteur du discours rapporté (Hodge, 2013). Dans tous les cas, comme dans l'exemple 7, la partie répétée a besoin de l'unité qu'elle encadre pour faire sens dans le discours.

(7)

PT:1 RÉFLÉCHIR QUOI PT:DET ONCLE DONNER BIEN SOUVENIR QUOI RÉFLÉCHIR

'Je réfléchis aux bons souvenirs que mon oncle m'a laissés.'

(CLSFB_03_06, S007, 08:25.500-08:32.500)

\subsubsection{Fonctions sémantiques}

Au sein du domaine sémantique, on distingue trois sous-domaines. Le premier regroupe les répétitions qui interviennent dans la cohésion du propos ; le deuxième couvre celles qui sont porteuses d'une précision sémantique, ou spécification; le troisième regroupe les répétitions qui interviennent dans l'organisation chronologique des évènements. Les différentes fonctions liées à ces trois sous-domaines sont présentées dans le tableau 3 avec leur fréquence d'apparition.

Tableau 3. - Typologie fonctionnelle - Domaine sémantique.

\begin{tabular}{|c|c|}
\hline \multicolumn{2}{|l|}{ Domaine sémantique } \\
\hline Sous-domaines et fonctions & Nombre d'occurrences \\
\hline Cohésion & 255 \\
\hline$<\mathrm{S}$ : Cohésion Thème> & 173 \\
\hline$<\mathrm{S}:$ Cohésion Agent> & 58 \\
\hline <S: Cohésion Personnage> & 13 \\
\hline$<\mathrm{S}$ : Développement> & 11 \\
\hline Spécification & 59 \\
\hline <S : Spécification> & 59 \\
\hline Chronologie & 21 \\
\hline$<\mathrm{S}:$ Causalité $>$ & 7 \\
\hline$<\mathrm{S}:$ Simultanéité> & 5 \\
\hline <S : Redondance> & 5 \\
\hline$<\mathrm{S}:$ Succession $>$ & 4 \\
\hline Total & 335 \\
\hline
\end{tabular}


Parmi les trois sous-domaines identifiés, les fonctions de cohésion sont les plus représentées. Des quatre fonctions identifiées, seules les deux premières (cohésion par la reprise du thème: plus de $60 \%$; cohésion par la reprise de l'agent: $20 \%$ ) sont présentes chez l'ensemble des signeurs. Dans l'exemple 6 ci-dessus, la répétition du signe COCA (celle dont les deux constituants sont mis en italique) ne remplit aucune autre fonction que celle de supporter la cohésion du propos en en rappelant le sujet. L'exemple 8 illustre un cas de répétition de la référence à l'agent. La signeuse parle de son expérience personnelle et ne cesse de rappeler par l'emploi du pronom de première personne (PT:1) qu'elle parle d'elle-même. Cette répétition est d'autant plus emphatique que la marque de la première personne n'est pas obligatoire lorsqu'elle est déductible du contexte.

(8)

PT:1 SUIVRE ADAPTER PT:1 LS ADAPTER STYLE PT:1 GRAND PT:1 AMI LS RENCONTRER JAMAIS PT:6 PT:1 LS COMPRENDRE RIEN PT:1 SAVOIR-PAS PT:1 HABITUDE ÉQUIPE PT:1 CHANCE PT:1 OUVRIR-ESPRIT PT:1 RENCONTRER PT:1 RENCONTRER

'Je suis les autres et je m'adapte. J'adapte ma façon de signer en fonction du style des autres. Quand j'étais jeune, j'étais habituée à signer avec le même groupe d'amis et à ne jamais rencontrer les autres. Je ne comprenais rien à leurs signes, je ne les connaissais pas. J'étais habituée à mon groupe d'amis. Mais heureusement je suis ouverte d'esprit. J'ai rencontré une personne, puis beaucoup d'autres.'

(CLSFB_33_14, S067, 03:41.200-03:56.400) représentée, à savoir l'expression de la causalité, est illustrée dans l'exemple 9.

(9)

SAVOIR PT:3 DEUX AUTRE DIFFÉRENT LS RICHE FUTUR RENCONTRE SOURD PT:POSS NAMUR LIÈGE PT:3 SAVOIR DIFFÉRENT LS RICHE

'Elle sait que connaitre deux langues des signes différentes est une richesse parce que, dans le futur, elle sera amenée à rencontrer des personnes sourdes originaires de Namur ou de Liège.'

(CLSFB_33_14, S068, 01:40-01:47.400)

\subsubsection{Fonctions pragmatiques}

Pour le domaine pragmatique, les fonctions ont été classées en quatre sous-domaines (cf. tableau 4): les fonctions liées à la mise en relief des informations, celles qui relèvent de la gestion de la formulation en temps réel, celles qui sont liées à l'interaction, et celles qui relèvent de l'organisation de l'énonciation.

Tableau 4. - Typologie fonctionnelle - Domaine pragmatique.

\begin{tabular}{|l|l|}
\hline \multicolumn{2}{|l|}{ Domaine pragmatique } \\
\hline Sous-domaines et fonctions & Nombre d'occurrences \\
\hline Mise en relief & 419 \\
\hline
\end{tabular}




\begin{tabular}{|c|c|}
\hline$<\mathrm{P}:$ Parallélisme $>$ & 170 \\
\hline$<\mathrm{P}:$ Emphase Position Signeur $>$ & 55 \\
\hline$<\mathrm{P}:$ Changement Point de Vue $>$ & 39 \\
\hline$<\mathrm{P}:$ Conclusion $>$ & 37 \\
\hline$<\mathrm{P}$ : Mise au premier plan $>$ & 29 \\
\hline$<\mathrm{P}:$ Encadrement Unité Thématique $>$ & 29 \\
\hline$<\mathrm{P}:$ Encadrement Contraste $>$ & 28 \\
\hline$<\mathrm{P}:$ Encadrement Rhème> & 17 \\
\hline$<\mathrm{P}:$ Gradation Position Signeur $>$ & 15 \\
\hline Formulation en temps réel & 115 \\
\hline$<\mathrm{P}:$ Réparation $>$ & 51 \\
\hline$<\mathrm{P}:$ Parenthèse $>$ & 41 \\
\hline$<\mathrm{P}:$ Recherche Lexicale $>$ & 22 \\
\hline$<\mathrm{P}:$ Suppression $>$ & 1 \\
\hline Interaction & 73 \\
\hline$<\mathrm{P}:$ Transition $>$ & 28 \\
\hline$<\mathrm{P}:$ Phatique $>$ & 24 \\
\hline$<\mathrm{P}:$ Chevauchement $>$ & 16 \\
\hline$<\mathrm{P}:$ Convention Lexicale $>$ & 3 \\
\hline$<\mathrm{P}$ : Métalinguistique $>$ & 2 \\
\hline Organisation & 71 \\
\hline$<\mathrm{P}:$ Ponctuation $>$ & 62 \\
\hline$<\mathrm{P}$ : Énumération $>$ & 9 \\
\hline Total & 678 \\
\hline
\end{tabular}

Les répétitions relevant du domaine pragmatique sont sensiblement plus fréquentes (14,23/100 signes) que les répétitions grammaticales (5,25/100 signes) et sémantiques (7,01/100 signes). C'est essentiellement le sous-domaine de la "mise en relief des informations " qui provoque cet écart (8,87/100 signes). La fonction étiquetée de " parallélisme » y est la plus fréquente $(3,58 / 100$ signes) : la répétition rapproche deux 
éléments pour souligner leur similitude, leur différence ou leur opposition (Tannen, 2007). Dans l'exemple 10, la répétition de la séquence de signes PARENTS ENFANT LS apporte un cadre identique pour mettre en contraste deux termes, à savoir SOURD et ENTENDANT ${ }^{9}$.

(10)

PARENTS ENTENDANT UN ENFANT LS LS LS PARENTS SOURD ENFANT SOURD LS DIFFÉRENT

'Les parents entendants signent à leur enfant différemment des parents sourds visà-vis de leur enfant sourd.'

(CLSFB_33_14, S067, 02:18.600-02:26.400)

Les fonctions liées à la " gestion de la formulation du propos en temps réel » sont liées au processus d'hésitation et de réparation de la chaine signée. Les données contiennent peu d'exemples de cette fonction, c'est-à-dire, selon le principe méthodologique énoncé supra, peu de répétitions dont la seule fonction possible relève de l'hésitation et de la réparation (2,31/100 signes). Dans l'exemple 11, le signeur hésite sur son âge à l'époque de l'évènement dont il parle. D'abord, il s'interrompt dans la production de son signe, ensuite, il produit le signe TRENTE-HUIT en détournant la tête, avant de répéter le signe pour valider la recherche lexicale ${ }^{10}$.

(11)

PT:1 [troncation du signe TRENTE-HUIT] COMMENCER CRÉER PT:3 TRENTE-HUIT

TRENTE-HUIT ÂGE PT:1 TRENTE-HUIT

'J'avais... Quand ils ont commencé à mettre sur pied [la compétition], j'avais trente-

huit... trente-huit, j'avais trente-huit ans.'

(CLSFB_03_06, S008, 10:57-11:01.500)

31 Étant donné que la sélection des données s'est faite en privilégiant les longs passages monologiques au sein des conversations, le sous-domaine des fonctions liées à l'interaction se trouve logiquement faiblement représenté. La fonction de transition, en début de tour de parole, permet au signeur de prendre sa place, ou de reprendre un tour interrompu par l'intersigneur, comme dans l'exemple 12.

(12)

SUD CHAUD LS BIEN CHAUD SUD NORD [courte interruption de l'intersigneur] SUD CHAUD LS NORD FROID

'Au Sud, la langue de signes est plus chaude, alors qu'au Nord [courte interruption de l'intersigneur] Au Sud, la langue des signes est plus chaude, alors qu'au Nord elle est plus froide.'

(CLSFB_37_14, S075, 01:41-01:48.400)

Enfin, le sous-domaine des fonctions pragmatiques liées à l'organisation du discours comprend deux types de répétitions: celles qui ponctuent le discours et celles, beaucoup moins fréquentes, qui offrent un cadre à l'énumération. Dans le cas de l'énumération, la répétition d'un terme dans chaque élément de la liste produit la structure énumérative et le lien entre les éléments listés. Les répétitions ont été identifiées comme ponctuantes lorsque les signes répétés jouent le rôle d'un stimulant pour faire progresser la pensée, d'une impulsion qui introduit de nouvelles informations ou de nouveaux exemples. Dans l'exemple 13, le signeur introduit un élément d'information supplémentaire à son propos à l'aide de chacune des trois occurrences du signe AUSSI. Par ailleurs, la répétition du signe ÉVIDENT relève de l'emphase de la position du signeur (sous-domaine de la « mise en relief », cf. supra).

(13)

OUI OUI ÉVIDENT ÉVIDENT FIN EXPRESSION DIVERS EXPRESSION REMARQUER FINI AUSSI LS VITE LENT ÉVIDENT EXPRESSION ÉVIDENT TOUT AUSSI COULEUR 


\author{
VISAGE TEMPÉRATURE NIVEAU CLAIR FONCÉ AUSSI COULEUR DIFFÉRENT \\ 'Oui, c'est évident, c'est évident. Les expressions du visage sont fines et variées. On \\ les remarque directement. De plus, il y a la manière de signer, rapide ou lente. C'est \\ évident. L'expression des sentiments est évidente en général. De plus, il y a les \\ nuances de la teinte du visage qui s'éclaircit ou s'assombrit. De plus, les teintes \\ peuvent être différentes.' \\ (CLSFB_03_06, S008, 00:430-00:49)
}

Une petite proportion des répétitions observées (8\%) n'a pu être associée à aucun des trois domaines présentés ci-dessus. Par exemple, un signeur produit deux fois le signe FINI à 5 secondes d'intervalle: il explique qu'une blessure au coude l'a empêché de courir à vélo pendant un an, mais qu'une fois la blessure soignée (SOIGNER ALLERMIEUX FINI), il s'est entrainé et a réussi à obtenir la troisième place en compétition (REUSSIR FINI PT:PRO1 TROISIEME) (CLSFB_03_06, S008, 11:28-11:33). Aucune fonction particulière ne semble pouvoir être attribuée à ce type de cas. Cependant, étant donné que le parti pris de cette étude était de relever toutes les répétitions apparaissant au sein d'un tour de parole, les signes répétés sans raison apparente ont été pris en compte dans le recensement et ont été étiquetés « autres ».

\title{
6. La répétition et la conversation
}

La répétition est un processus qui s'apparente à l'automaticité et de ce fait soulage l'effort de production et de réception de la parole. Son usage est perçu positivement pour l'aisance qu'il procure tant au locuteur qu'à l'interlocuteur (Tannen, 2007; Götz, 2013). Selon Tannen, la maitrise des différents emplois de la répétition varierait en fonction de la culture dans laquelle un individu s'inscrit. Au sein d'une même culture, les variations individuelles s'exprimeraient dans les limites d'un usage partagé. La présente analyse en LSFB confirme cette hypothèse.

La répétition est inhérente à la prise de parole des signeurs. Tous les tours de parole contiennent des répétitions, et leur nombre augmente avec la longueur du tour. La répétition aide à la construction des tours et sert de charpente à la construction du sens. En moyenne, les signeurs produisent 28,65 répétitions tous les 100 signes ; un peu plus d'un tiers des signes $(37,13 \%)$ d'un discours consiste en une réutilisation du même matériel lexical. Chaque tour de parole contient au moins une répétition non contigüe et, en moyenne, cette forme est la plus fréquente (62\%).

Quelle que soit la forme de la répétition, elle n'implique généralement qu'un seul répété $(88,18 \%)$. Ce sont les signes lexicaux qui sont principalement touchés par la répétition $(67 \%)$, suivis des signes pointés $(11 \%)$ et des signes partiellement lexicalisés (Johnston \& Schembri, 2010), ce qui correspond à la répartition habituelle des différents types de signes dans un discours en général (Hodge, 2013). Par contre, alors que dans les LV les répétitions portent majoritairement sur les mots-outils, les 22 signes les plus répétés chez chacun des signeurs étudiés comptent pour moitié des signes pleins et pour moitié des signes-outils (Notarrigo, 2017, p. 276-278).

Si les répétitions grammaticales, c'est-à-dire participant au fonctionnement morphosyntaxique de la langue, sont celles qui ont été le plus décrites dans la littérature sur les LS, elles ne constituent qu'une portion limitée (18\%) des occurrences rencontrées en conversation. En effet, la prise en compte de données dépassant le cadre de l'énoncé et s'étendant à de larges pans de discours spontanés met en lumière que les répétitions 
les plus fréquentes sont celles qui remplissent une fonction pragmatique $(49,5 \%)$, suivies de celles qui ont une fonction sémantique $(24,5 \%)$.

Que ce soit pour leur fréquence, pour les formes qu'elles prennent (contigües, non contigües, encadrantes), pour les fonctions qu'elles remplissent, tous les signeurs considérés semblent avoir acquis un même style d'utilisation grammatical, sémantique et pragmatique de la répétition en discours. De façon surprenante, étant donné le caractère non préparé des productions étudiées, très peu des répétitions rencontrées sont liées au phénomène d'hésitation (2,31/100 signes). Au contraire, les signeurs montrent une grande capacité à imbriquer les répétitions les unes avec les autres. L'usage de la répétition confère à leurs discours un style commun dépassant les seules variations individuelles. Le prolongement de cette étude par une comparaison avec des données de narration, d'explication et d'argumentation permettra de mieux comprendre comment la variété des genres discursifs se manifeste, en LSFB, entre identité stylistique et variation individuelle.

Par ailleurs, cette étude amène à se demander si la fréquence et la variété des fonctions des répétitions observées est spécifique aux LS (lié à la modalité visuo-gestuelle et à la façon dont celle-ci contraint la production et la perception linguistique), ou s'il s'agit plus généralement d'une propriété de la conversation orale (liée aux échanges non préparés et perçus en temps réel). Une étude contrastive des usages de la répétition dans des données comparables en français oral et en LSFB nous permettra d'amener des éléments de réponse à cette question.

\section{BIBLIOGRAPHIE}

BERGMAN, Brita \& DAHL, Osten. (1994). Ideophones in Sign Language? The Place of Reduplication in the Tense-Aspect System of Swedish Sign Language. Dans C. Bache, H. Basboll \& C. Lindberg (dir.), Tense, Aspect and Action. Empirical and Theoretical Contributions to Language Typology (p. 397-422). New York : Mouton de Gruyter.

BöRSTELL, Carl. (2011). Revisiting Reduplication: Toward a Descritpion of Reduplication in Predicative Signs in Swedish Sign Language (Mémoire de master). Stockholms Universitet, Stocholm.

Branchini, Chiara, Cardinaletti, Anna, Cecchetto, Carlo, Donati, Caterina \& Geaci, Carlo. (2013). Wh-duplication in Italian Sign Language (LIS). Sign Language and Linguistics, 16(2), 157-188. <https://doi.org/10.1075/sll.16.2.03bra>.

CANDEA, Maria. (2000). Contribution à l'étude des pauses silencieuses et des phénomènes dits "d'hésitation » en français oral spontané. Étude sur un corpus de récits en classe de français (Thèse de doctorat). Université Sorbonne Nouvelle - Paris 3, Paris.

CRASBORN, Onno \& SLOETJES, Han. (2008). Enhanced ELAN Functionality for Sign Language Corpora. In 6th International Conference on Language Resources and Evaluation (LREC 2008) / 3rd Workshop on the Representation and Processing of Sign Languages: Construction and Exploitation of Sign Language Corpora (p. 39-43). 
CRASBORn, Onno, VAn DER KooIJ, Els, Ros, Johan \& DE Hoop, Helen. (2009). Topic Agreement in NGT (Sign Language of the Netherlands). The Linguistic Review, 26, 355-370.

CRIBLE, Ludivine, Dumont, Amandine, Grosman, Iulia \& NotARRIGO, Ingrid. (2019). (Dis)fluency across Spoken and Signed Languages: Application of an Interoperable Annotation Scheme. In L. Degand, G. Gilquin, L. Meurant \& A. C. Simon (dir.), Fluency and Disfluency across Languages and Language Varieties (p. 17-39). Louvain-la-Neuve : Presses universitaires de Louvain.

DISTER, Anne. (2007). De la transcription à l'étiquetage morphosyntaxique. Le cas de la banque de données textuelle orale VALIBEL (Thèse de doctorat). Université catholique de Louvain, Louvain-la-Neuve.

Dovicchi, Francesca. (2010). Costrutti-eco nell'italiano parlato. Da repetizione a cardinalità. Tübingen : Narr Francke Attempto Verlag.

FISCHER, Susan \& JANIS, Wynne. (1990). Verb Sandwiches in American Sign Language. Current Trends in European Sign Language Research, 9, 279-293.

FILIPCZAK, Joanna \& MostowsKI, Piotr. (2013). Repetition in Polish Sign Language (PJM). Discourse Grammar - Information Structure? Poster présenté à la conférence « Theoretical Issues in Sign Language Research (TISLR) $11 »$, Londres, Royaume-Uni.

FRÉDÉRIC, Madeleine. (1985). La répétition. Étude linguistique et rhétorique. Tubingen : Niemeyer. GöTZ, Sandra. (2013). Fluency in Native and Nonnative English Speech. Amsterdam : John Benjamins Publishing Company.

GABARRó-LÓPEZ, Silvia. (2017). Discourse Markers in French Belgian Sign Language (LSFB) and Catalan Sign Language (LSC): BUOYS, PALM-UP and SAME. Variation, Functions and Position in Discourse (Thèse de doctorat). Université de Namur, Namur.

GabarRó-LóPEZ, Silvia \& MEURANT, Laurence. (2014). The Use of Buoys across Genres in French Belgian Sign Language (LSFB). Dans Actes du IXe colloque de linguistique des doctorants et jeunes chercheurs du Laboratoire MoDyCo (COLDOC 2013) : La question des genres à l'écrit et à l'oral (p. 43-54). Paris : Université Paris-Ouest.

HERBERT, Marjorie. (2012). Pluralization in German Sign Language (Mémoire de Bachelier). Swarthmore College. Disponible en ligne sur <https://scholarship.tricolib.brynmawr.edu/ handle/10066/10110>.

HoHENBERger, Annette \& LeUninger, Helen. (2012). Production. Dans R. Pfau, M. Steinbach \& B. Woll (dir.), Sign Language. An International Handbook (chap. 30, p. 711-738). Berlin/Boston : De Gruyter Mouton.

HODGE, Gabrielle. (2013). Patterns from a Signed Language Corpus: Clause-Like Units in Auslan (Australian Sign Language) (Thèse de doctorat). Macquarie University, Sydney.

HoRlacher, Anne-Sylvie \& DoeHler, Simona Pekarek. (2014). Pivotage in French Talk-inInteraction: On the Emergent Nature of [clause-NP-clause] Pivots. Pragmatics, 24(3), 593-622.

JOHNSTON, Trevor \& SCHEMBRI, Adam. (2010). Variation, Lexicalization and Grammaticalization in Signed Languages. Language, 131, 19-35.

Kendon, Adam. (2004). Gesture: Visible Action As Utterance. Cambridge : Cambridge University Press. Kimmelman, Vadim. (2014). Information Structure in Russian Sign Language and Sign Language of the Netherlands (Thèse de doctorat). Universiteit van Amsterdam, Amsterdam.

KLIMA, Edward S. \& BeLLUGI, Ursula. (1979). The Signs of Language. Cambridge, MA : Harvard University Press. 
LIDDELL, Scott K. (2003). Grammar, Gesture and Meaning in American Sign Language. Cambridge : Cambridge University Press.

MEURANT, Laurence. (2008). Le regard en langue des signes. Anaphore en langue des signes de Belgique francophone (LSFB). Morphologie, syntaxe, énonciation. Rennes : Presses universitaires de Rennes.

MEURANT, Laurence. (2010). Simultanéité et linéarité : leur grammaticalisation en langue des signes française de Belgique (LSFB). Dans C. Douay (dir.), Système et chronologie (p. 255-273). Rennes : Presses universitaires de Rennes.

MEURANT, Laurence. (2015). Corpus LSFB. Un corpus informatisé en libre accès de vidéos et d'annotations de la langue des signes de Belgique francophone (LSFB). Laboratoire de Langue des signes de Belgique francophone (LSFB-Lab). FRS-F.N.R.S. et Université de Namur. Disponible en ligne sur $<$ www.corpus-lsfb.be>.

MEURANT, Laurence \& SinTE, Aurélie. (2016). La reformulation en langue des signes de Belgique francophone (LSFB) : analyse dans un corpus de trois types de discours. L'information grammaticale, $149,32-44$.

Nicodemus, Brenda. (2011). Disfluencies in American Sign Language and English. Communication présentée au « 33rd Annual Conference of the German Linguistic Society (DGfS) », Georg August University, Göttingen.

NoRÉN, Niklas. (2007). Apokoinou in Swedish Talk-in-Interaction: A Family of Methods for Grammatical Construction and the Resolving of Local Communicative Projects (Thèse de doctorat). Linköping University Electronic Press.

NOTARRIGO, Ingrid. (2017). Marqueurs de (dis)fluence en langue des signes de Belgique francophone (Thèse de doctorat). Université de Namur, Namur.

NotARRIGo, Ingrid, MEURANT, Laurence \& SIMON Anne Catherine. (2016). Repetitions of Signs according to Language Background: A Comparative Analysis between Native, Near-Native and Late Signers. Poster présenté au « 12th International Conference on Theoretical Issues in Sign Language Research », Melbourne.

PfAU, Roland \& STEINBACH, Markus. (2006), Pluralization in Sign and in Speech: A Cross-Modal Typological Study. Linguistic Typology, 10, 135-182.

RISLER, Annie. (2014). Parenthèses et ruptures énonciatives en langue des signes française. Discours, 14, 1-19.

SCHEUTZ, Hannes. (2005). Pivot Constructions in Spoken German. Dans A. Hakulinen \& M. Selting (dir.), Syntax and Lexis in Conversation (p. 103-128). Amsterdam : John Benjamins Publishing Company.

SHRIBERG, Elizabeth E. (1994). Preliminaries to a Theory of Speech Disfluencies (Thèse de doctorat). University of California, Berkeley.

TANNEN, Deborah. (2007). Talking Voices. Repetition, Dialogue, and Imagery in Conversational Discourse ( 2 éd.). Cambridge : Cambridge University Press.

VAN LOON, Esther. (2012). What's in the Palm of Your Hands? Discourse Functions of Palm-Up in Sign Language of the Netherlands (Mémoire de master). Universiteit van Amsterdam, Amsterdam. Vermeerbergen, Myriam \& De VRIendT, Sera. (1994). The Repetition of Signs in Flemish Sign Language. Dans I. Ahlgren, B. Bergman \& M. Brennan (dir.), Perspectives on Sign Language Usage 
[Papers from The Fifth International Symposium on Sign Language Research] (pp. 201-214). University of Durham.

\section{NOTES}

1. Accord sur le nombre de répétitions: $\mathrm{k}=0,7504$; accord sur la forme des répétitions : $\mathrm{k}=0,8661$; accord sur le domaine de fonction: $\mathrm{k}=0,5140$. Les divergences entre annotateurs concernent surtout l'identification des fonctions au sein d'un domaine. Le choix fait ici de n'attribuer qu'une seule fonction par répétition occulte leur caractère multifonctionnel, tout en diminuant le taux d'accord entre annotateurs. Par ailleurs, vu les divergences récurrentes qu'elles suscitent, cinq catégories de fonctions semblent devoir être plus explicitement définies ou mieux délimitées : il s'agit de $<\mathrm{G}$ : Syntagme $>,<\mathrm{S}$ : CohésionAgent $>,<\mathrm{S}$ : Développement $>,<\mathrm{P}$ : ChangementPointDeVue $>,<\mathrm{P}$ : Parallélisme>, présentées ci-dessous. Pour une discussion plus détaillée des résultats du test inter-annotateurs, voir Notarrigo (2017, p. 260-263).

2. Toutefois, si un répétable et un répété partagent la même articulation manuelle, mais sont accompagnés d'une articulation labiale différente, le bloc n'est pas validé en tant que répétition. Nous considérons ces cas comme des signes distincts, l'articulation labiale (calquant un mot de la LV) véhiculant un sens différent d'un signe à l'autre.

3. Les 18 signeurs représentent trois profils linguistiques définis en fonction de l'âge d'acquisition de la LSFB : 6 natifs (acquisition en famille dès la naissance, au contact de parents sourds signants), 6 quasi natifs (acquisition avant 7 ans) et 6 tardifs (acquisition après 7 ans). Aucune différence significative n'a pu être établie entre ces groupes du point de vue de l'usage des répétitions, c'est pourquoi les 18 signeurs sont considérés ici ensemble.

4. L'ensemble des tâches se trouve détaillé sur le site du corpus : <www.corpus-LSFB.be>.

5. Les tours comportant de courtes interruptions qui n'amènent pas un changement de tour de parole ont été conservés. Ce sont les moments d'échanges dans lesquels les interlocuteurs prennent alternativement la parole qui ont été écartés.

6. Le logiciel ELAN est développé par le Max Planck Institute for Psycholinguistics, The Language Archive, Nijmegen, The Netherlands (<https://tla.mpi.nl/tools/tla-tools/elan/>).

7. Pour faciliter la lecture, les exemples ont été transcrits. Ces transcriptions se limitent à rendre la succession des signes manuels, identifiés par une glose en majuscules. Les gloses PT suivies d'un chiffre désignent des signes pointés ayant une valeur de pronom personnel ; PT:POSS et $P T: D E T$ désignent respectivement un signe pointé à valeur sémantique de possessif ou de déterminant. Les éléments non manuels, le rythme et les pauses, qui jouent pourtant un rôle important dans l'identification des répétitions (notamment pour la distinction entre les RN et les RNe), n'y apparaissent pas. Le lecteur est encouragé à visionner les extraits sur le site du Corpus LSFB, en suivant les références entre parenthèses données à la fin de chaque exemple. Pour ce faire, il lui suffit de se créer un compte (gratuit) à l'adresse <www.corpus-lsfb.be> et de faire une recherche «libre». Dans la référence de type "CLSFB_AA_BB, SXXX, 00:00.000-00:01.900 », AA renvoie au numéro de la session, BB au numéro de la tâche, XXX au numéro du signeur, le tout étant suivi du code temporel de début et de fin de l'extrait considéré.

8. La mesure par 100 signes a été préférée à une mesure par minute afin d'éviter l'influence de la variable de la vitesse de signation sur les résultats (les signeurs natifs étant, selon les données de cette étude, plus rapides que les signeurs quasi natifs et tardifs).

9. La répétition du signe LS (LANGUE-DES-SIGNES) a été identifiée comme une répétition grammaticale de durée.

10. La troisième et dernière occurrence du signe TRENTE-HUIT de l'extrait a été analysée comme une répétition encadrante à valeur de mise au premier plan (sous-domaine de la «mise en relief »), dont le répétable est la deuxième occurrence (en italique). 


\section{RÉSUMÉS}

Cette étude décrit l'usage des répétitions en conversation spontanée chez les locuteurs de la langue des signes de Belgique francophone (LSFB). L'approche suivie consiste à prendre en compte sans à priori toutes les répétitions rencontrées dans les productions de 18 signeurs du Corpus $L S F B$, de façon à disposer d'un premier aperçu global de la façon dont la répétition contribue à la structuration grammaticale, sémantique et pragmatique du discours. L'un des apports du travail tient à la typologie formelle et fonctionnelle qu'il propose. Les résultats montrent que les signeurs font un usage similaire des répétitions, ce qui confère à leurs discours un style commun.

This study aims at describing the use of repetitions in spontaneous conversation in French Belgian Sign Language (LSFB). All occurrences of repetitions are taken into account in the productions of 18 signers taken from the Corpus LSFB, in order to provide as complete an overview as possible on how repetition contributes respectively to the grammatical, the semantic and the pragmatic level of discourse structure. One of the contribution of this work lies in the formal and functional typology of repetitions that it offers. The results show that all signers make a similar use of repetitions, which provides a common style to their discourses.

\section{INDEX}

Keywords : sign languages, repetition, typology, forms, functions, grammar, semantics, pragmatics

Mots-clés : langue des signes, répétition, typologie, formes, fonctions, grammaire, sémantique, pragmatique

\section{AUTEURS}

\section{INGRID NOTARRIGO}

École \& Surdité, Sainte-Marie Namur

\section{LAURENCE MEURANT}

FRS-FNRS, NaLTT, Université de Namur 